\title{
Initial Clinical Experience Treating Patients With Lung Cancer on a 6MV-Flattening-Filter-Free 0- Ring Linear Accelerator
}

Andrew R. Barsky ${ }^{1}$, Hui Lin ${ }^{1}$, Amberly Mendes ${ }^{1}$, Alexandra Dreyfuss ${ }^{2}$, Christopher Wright ${ }^{1}$, Emily $\mathrm{I}$ Anstadt ${ }^{1}$, Abigail T. Berman ${ }^{1}$, William P. Levin ${ }^{1}$, Keith A. Cengel ${ }^{1}$, Nathan Anderson ${ }^{1}$, Lei Dong ${ }^{1}$, James M. Metz ${ }^{1}$, Taoran $\mathrm{Li}^{1}$, Steven Feigenberg ${ }^{1}$

1. Department of Radiation Oncology, Hospital of the University of Pennsylvania, Philadelphia, USA 2. Department of Radiation Oncology, Memorial Sloan Kettering Cancer Center, New York, USA

Corresponding author: Andrew R. Barsky, andrew.barsky@uphs.upenn.edu

\section{Abstract \\ Introduction}

Modern technologies, like intensity-modulated radiotherapy (IMRT) and volumetric-modulated arc therapy (VMAT), have improved the therapeutic ratio of thoracic radiotherapy (TRT) for lung cancer (LC). Halcyon ${ }^{\mathrm{TM}}$ (Varian Medical Systems, Palo Alto, CA, USA), a novel 6MV-flattening-filter-free O-ring linear accelerator (6X-FFF ORL), was designed to deliver IMRT and VMAT with greater speed than a C-arm linac. Herein, we report our initial clinical experience treating patients with LC on this linac.

\section{Methods}

All patients who received TRT for LC on the 6X-FFF ORL at our institution were retrospectively identified. Patients' clinicopathologic data, radiotherapy details, early disease-control and toxicity outcomes, dosimetric data, couch corrections, and treatment times are reported.

\section{Results}

Between 10/2018-12/2019, 30 consecutive patients (median age 66 years, range 54-94 years) received definitive or post-operative TRT for LC (median 66 Gy/33 fractions; range 5-70 Gy/2-37 fractions) following four-dimensional computed tomography (CT) simulation (97\%) using daily kilovoltage KV cone-beam CT (CBCT) (100\%) on a 6X-FFF ORL for non-small cell LC (84\%) or small cell LC (16\%), with 53\% receiving VMAT, $43 \%$ receiving static-field IMRT, and 77\% receiving concurrent systemic therapy. All plans were approved through institutional peer review. The average three-dimensional vector couch correction based on CBCT guidance was $0.90 \pm 0.50 \mathrm{~cm}$. The average beam-on and beam on plus CBCT times were $1.7 \pm 1.1$ $\mathrm{min}$, and $5.0 \pm 3.2 \mathrm{~min}$, respectively. Grade 3 dyspnea and fatigue occurred in $3 \%$ and $3 \%$ of patients, respectively. There were no grade $\geqslant 4$ toxicities.

Received 08/21/2020

Review began 08/27/2020 Review ended 09/02/2020 Published 09/09/2020

\section{() Copyright 2020}

Barsky et al. This is an open access

article distributed under the terms of the Creative Commons Attribution License CC-BY 4.0., which permits unrestricted use, distribution, and reproduction in any medium, provided the original author and source are credited.

\section{Conclusion}

In this first clinical report of TRT for LC on a 6X-FFF ORL, daily CBCT-guided treatment was fast and safe with respect to dosimetry and clinical outcomes. Thus, use of this linac for TRT may increase LC patient throughput without a detriment in radiotherapy quality.

Categories: Medical Physics, Radiation Oncology, Oncology

Keywords: lung cancer, non-small cell lung cancer, small cell lung cancer, radiotherapy, imrt, vmat, flattening filter free, radiation, throughput

\section{Introduction}

Clinical management of primary lung cancer (LC) is guided by pathologic evaluation, staging, comorbidities, multidisciplinary discussion, and shared decision-making with the patient $[1,2]$. Patients who present with early-stage disease [3,4], locally-advanced disease [5-7], distant metastatic disease [8,9], or even recurrent disease [10], may have a National Comprehensive Cancer Network (NCCN) guideline-supported role for thoracic radiotherapy (TRT) as a definitive, neoadjuvant, and/or adjuvant treatment modality, with or without systemic therapy $[1,2]$.

Technological advances in radiotherapy (RT) delivery have improved the therapeutic ratio of TRT, such that NCCN deems computed tomography (CT)-planned three-dimensional conformal RT (3D-CRT) a minimum technologic standard. NCCN also states that use of more advanced technologies, including intensitymodulated radiotherapy (IMRT) and volumetric-modulated arc therapy (VMAT), is appropriate to deliver RT safely [1]. IMRT and VMAT utilize physician-outlined "contours" of CT-based oncologic target tissues and critical organs-at-risk (OARs) to generate RT dose distributions that maximize target coverage, minimize 
OAR doses, and optimally, decrease toxicity. IMRT has been shown to reduce high-grade RT pneumonitis and cardiac dose in patients receiving TRT for Stage III non-small cell LC (NSCLC) compared to 3D-CRT, with comparable survival and disease-control, suggesting its role as a standard of care [11]. VMAT, a subtype of IMRT, is delivered in dynamic, continuous arcs that allow for reduced RT treatment times compared to static-field IMRT [12]. Further, static-field IMRT and VMAT may be administered using a flattening-filterfree (FFF) linear accelerator (linac), which can increase dose rate, and decrease head scatter and penumbra in comparison to flattening-filtered (FF) RT [13].

Halcyon $^{\mathrm{TM}}$ (Varian Medical Systems, Palo Alto, CA, USA), a novel 6 megavolt (MV)-flattening-filter-free (6XFFF) O-ring gantry linac, was built to administer RT with greater speed and throughput than a C-arm linac (CAL), with some similarities to TomoTherapy (Accuray, Sunnyvale, CA, USA) [14]. Published reports of 6XFFF O-ring linacs (ORL) are largely limited to pre-clinical planning studies, and have demonstrated similar plan quality with faster calculated treatment times for 6X-FFF ORLs versus CALs [14-19]. Reports of the clinical use of a 6X-FFF ORL to date have been limited to breast cancer [20], gynecologic cancers [12], and malignant pleural mesothelioma (MPM) [21], and have demonstrated fast treatment times and high throughput with the 6X-FFF ORL compared to CALs, with comparable plan quality, toxicity, and early disease-control.

In this report, we describe our initial clinical experience treating patients with TRT for LC on a 6X-FFF ORL. We hypothesized that dosimetry and treatment times for TRT on the 6X-FFF ORL would compare well with those of CALs.

\section{Materials And Methods}

In this IRB-approved, retrospective analysis, we reviewed all patients that received TRT on a 6X-FFF ORL for LC at our institution between 10/2018 and 12/2019. The only exclusion criterion included receiving one or more fraction of RT on a linac other than the 6X-FFF ORL. Electronic medical records were assessed for clinical, RT planning, treatment timing, and image-guided RT (IGRT) data.

\section{Prescriptions, constraints, and treatment planning}

Each patient was computed tomography (CT) simulated, in most cases with four-dimensional (4D)-CT, in the supine position, with a knee-foot lock and arm shuttle. A positron-emission tomography (PET)/CT, compression belt, and Vac-Lok ${ }^{\mathrm{TM}}$ bag (CIVCO Radiotherapy, Orange City, IA, USA) were used at the discretion of the treating physician.

For patients with intact disease or gross postoperative recurrence, a gross tumor volume (GTV) was contoured to encompass gross parenchymal and/or nodal disease identified on CT and/or PET, when applicable. An internal GTV (iGTV) was generated based upon all phases of the 4D-CT, to account for respiratory motion. The iGTV was uniformly expanded by $0.5-0.8 \mathrm{~cm}$ for parenchymal disease and $0.3-0.8 \mathrm{~cm}$ for nodal disease, while cropping out of uninvolved organs-at-risk (OARs), like heart, bone, and esophagus, to an internal target volume (ITV). Elective nodal volumes were not included. The ITV was uniformly expanded by $0.5 \mathrm{~cm}$ to a planning target volume (PTV). Patients were typically treated to 60-70 Gray (Gy) in $2 \mathrm{~Gy}$ fractions. In select cases, where hypofractionated courses were felt to be beneficial in order to expedite RT completion, patients were prescribed $30 \mathrm{~Gy}, 45 \mathrm{~Gy}, 55 \mathrm{~Gy}$, and $60 \mathrm{~Gy}$, in 10, 15, 22, and 15 fractions, respectively. For patients receiving postoperative RT (PORT) for mediastinal nodal disease, targets were contoured as per the Lung ART protocol [22], and were treated to 50 Gy in 25 fractions.

Treatment planning was completed in Eclipse ${ }^{\mathrm{TM}}$ (Varian Medical Systems, version 15.6) using 6MV FFF photon static-field IMRT or VMAT. The choice of static-field IMRT versus VMAT was at the discretion of the treating physician, and took into account patient-specific anatomy, as well as the ability to reduce low-dose radiation to the uninvolved lungs. Target coverage goals included that the dose received by $95 \%$ of the PTV (D95\%) be at least $95 \%$ of the prescription dose, and the PTV dose not exceed $110 \%$ of the prescription dose. OAR constraints included volume of lungs-iGTV receiving $\geqslant 20 \mathrm{~Gy}(\mathrm{~V} 20)<25 \%$ ( $\max <35 \%$ ), lungs-iGTV mean $<18$ Gy (max mean $<20 \mathrm{~Gy}$ ), esophagus V60<17\%, esophagus mean $<34 \mathrm{~Gy}$, heart dose receiving $0.03 \mathrm{cc}$ (D0.03cc) $<$ prescription dose, heart mean $<20 \mathrm{~Gy}$, spinal cord D0.03cc $<45 \mathrm{~Gy}$, and unilateral brachial plexus D0.03cc<66 Gy.

Single isocenter plans were generated for all patients. Planning used three to five arcs for VMAT, or four to six static fields or opposed static fields for IMRT, all with 6X-FFF energy and 800 monitor unit (MU)/minute dose rate. A dual-layer, $1.0 \mathrm{~cm}$ wide, stacked and staggered multi-leaf collimator (MLC) system was used for beam-modulation. Beam arrangement varied by technique. For all treatment plans, Photon Optimizer ${ }^{\mathrm{TM}}$ was used for optimization and the Analytical Anisotropic Algorithm (AAA) version 15.6 was used for dose calculation [23].

\section{Image guidance}

All patients received daily IGRT utilizing a kilovoltage $(\mathrm{kV})$ cone-beam CT (CBCT), as the 6X-FFF ORL does not perform $\mathrm{kV}$ planar imaging or have an optical distance indicator. Representative CT simulation and $\mathrm{kV}$ 


\section{Cureus}

CBCT images showing tumor regression during TRT are shown in Figure 1.

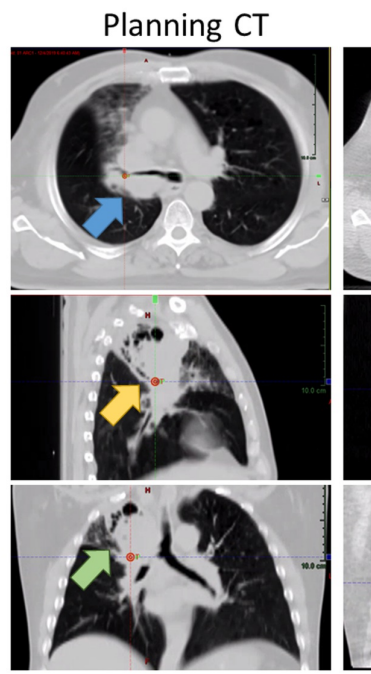

CBCT Fraction 1

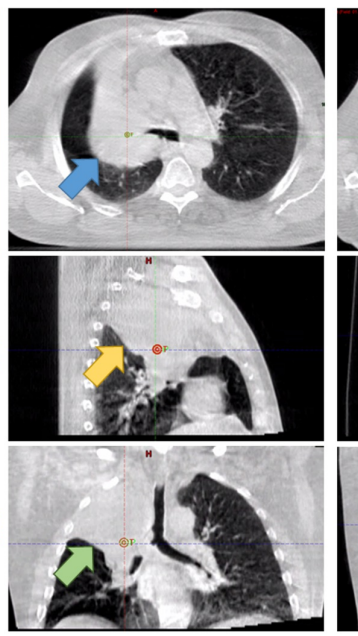

CBCT Fraction 30

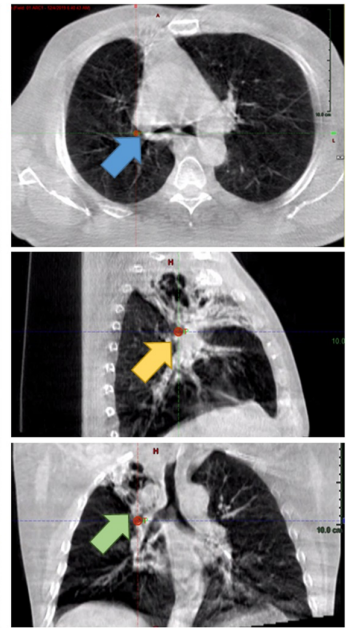

FIGURE 1: Representative computed tomography (CT) simulation and kilovoltage cone-beam CT (СВСТ) images

Representative computed tomography (CT) simulation images and corresponding 6MV flattening-filter-free O-ring linear accelerator (6X-FFF ORL) kV cone-beam CT images showing tumor regression in the axial (blue arrows), sagittal (yellow arrows), and coronal planes (green arrows) during thoracic radiotherapy.

\section{Data analysis}

Our primary aim was to describe the initial clinical evaluation of the use of a 6X-FFF ORL to deliver TRT to patients with LC, with respect to patient characteristics, treatment procedures, initial disease control, toxicity, dosimetry, couch corrections, and treatment speed. Disease-control was reported as clinical and/or radiographic local, regional, and distant control, and mortality. Common terminology criteria for adverse events (CTCAE) version 5.0 grading from weekly on-treatment and routine follow-up visits was used for acute toxicity. Plan dosimetry was assessed by target and OAR dose-volume histogram (DVH) review. Couch corrections, using daily CBCT match to bony anatomy, carina, or spinal cord from initial simulation, were calculated as the mean vector value for all TRT fractions for each patient. Treatment duration was assessed using the average beam-on time, treatment time (daily СBCT plus beam-on time), and total in-room time for each patient.

\section{Statistics}

Statistical analysis consisted of descriptive statistics (means, medians, ranges, standard deviations for continuous variables, and percentages for categorical variables). Couch correction and treatment speed data were compared qualitatively to reference values. SciPy 1.0 software was utilized for data analysis [24].

\section{Results}

\section{Patient clinico-pathologic and treatment characteristics}

We assessed 30 consecutive patients with a total of 31 primary tumors (one patient had bilateral primary tumors). The median age at RT and follow-up interval was 66 years (range, 54-94 years) and 11.9 months (range, 0.3-18.7 months), respectively.

Patients' LC histologies were NSCLC (84\%, n=26) and small-cell LC (SCLC) (16\%, n=5). TRT was delivered most commonly for Stage III disease $(52 \%, n=16$; Stage IV $16 \%, n=5$; Stage II $16 \%, n=5$; recurrent Stage III (rIII) 13\%, n=4; Stage I 3\%, n=1, treated fractionated because in a patient with synchronous Stage IIB disease). Patients with Stage IV disease were treated with TRT as local consolidative therapy $(80 \%, \mathrm{n}=4)$ or definitive therapy $(20 \%, n=1)$. Three patients (10\%) received PORT following lobectomy: two (67\%) for gross recurrence and one (33\%) for pathological N2 disease. Seventeen (57\%), six (20\%), and five (17\%) patients received concurrent and sequential, concurrent only, or sequential only systemic therapy with TRT, respectively. Concurrent systemic therapies consisted of carboplatin/paclitaxel $(57 \%, n=13)$, cisplatin/etoposide (17\%, $\mathrm{n}=4)$, carboplatin/pemetrexed $(13 \%, \mathrm{n}=3)$, carboplatin/nab-paclitaxel $(4 \%, \mathrm{n}=1)$, carboplatin/etoposide ( $4 \%, \mathrm{n}=1)$, and osimertinib (4\%, $\mathrm{n}=1)$. Sixteen $(53 \%)$ and two $(7 \%)$ received immunotherapy and targeted therapy, respectively, as part of their LC management. 


\section{Cureus}

Table 1 summarizes patients' RT details. All were treated supine (100\%, n=30) with VMAT (53\%, $n=16)$, static-field IMRT (43\%, n=13), or a combination (3\%, $n=1)$. Four-dimensional CT simulation was used for all but one patient $(97 \%, n=29)$, with compression belt $(70 \%, n=21)$, knee/foot lock and arm shuttle $(100 \%$, $\mathrm{n}=30)$, and Vac-Lok ${ }^{\mathrm{TM}}$ bag $(60 \%, \mathrm{n}=18)$. Primary tumors were most commonly right-sided $(70 \%, \mathrm{n}=21)$ and located in the right upper $(37 \%, \mathrm{n}=11)$ or right lower lobes $(30 \%, \mathrm{n}=9)$. TRT targets were most commonly lung, hilum, and mediastinum (55\%, $n=17)$, lung (16\%, $n=5)$, lung and hilum $(13 \%, n=4)$, or hilum and mediastinum (10\%, $\mathrm{n}=3$ ). The median TRT dose delivered was $66 \mathrm{~Gy}$ (range, 5-70 Gy), in 33 fractions (range, 2-37). Two patients (7\%) were planned to receive $55 \mathrm{~Gy}$ in 22 fractions and $70 \mathrm{~Gy}$ in 35 fractions, but did not complete their RT courses due to cancer-related mortality (one from hypoxemic respiratory failure due to a large malignant pleural effusion complicated by aspiration pneumonia, and one likely from a pulmonary embolism) following two and seven fractions, respectively. IGRT was daily kV CBCT for all patients (100\%, $\mathrm{n}=30)$.

\begin{tabular}{|c|c|}
\hline Variable & Value (\%) \\
\hline \multicolumn{2}{|l|}{ Laterality } \\
\hline Left & $8(27)$ \\
\hline Right & $21(70)$ \\
\hline Bilateral & $1(3)$ \\
\hline \multicolumn{2}{|l|}{ Lobe } \\
\hline Left upper lobe & 4 (13) \\
\hline Left lower lobe & $4(13)$ \\
\hline Right upper lobe & $11(37)$ \\
\hline Right middle lobe & 1 (3) \\
\hline Right lower lobe & $9(30)$ \\
\hline Left lower lobe and right lower lobe & $1(3)$ \\
\hline \multicolumn{2}{|l|}{ Target } \\
\hline Lung & $5^{\star}(16)$ \\
\hline Lung, hilum & $4(13)$ \\
\hline Lung, hilum, mediastinum & $17(55)$ \\
\hline Lung, hilum, mediastinum, supraclavicular fossa & $1(3)$ \\
\hline Hilum, mediastinum & $3(10)$ \\
\hline Hilum, mediastinum, bronchial stump & $1(3)$ \\
\hline \multicolumn{2}{|l|}{ Delivered dose (Gy) } \\
\hline Median & 66.0 \\
\hline Range & $5.0^{\circ}-70$ \\
\hline
\end{tabular}

\section{Number of fractions}

Median

Range

Modality

VMAT

Static-field

Both

Motion management 


\section{Cureus}

\section{Compression belt}

Yes

None

Immobilization

Knee/foot lock, arm shuttle

Vac-Lok ${ }^{T M}$ bag, knee/foot lock, arm shuttle

IGRT

kV CBCT

Systemic therapy

Concurrent only

Sequential only

Concurrent and sequential

None

\section{TABLE 1: Details of radiotherapy course}

Abbreviations: Gy, Gray; VMAT, volumetric-modulated arc therapy; 4D-CT, four-dimensional computed tomography; IGRT, image-guided radiotherapy; kV, kilovoltage; CBCT, cone-beam computed tomography; $\mathrm{RT}$, radiotherapy

* Values out of number of primary tumors, not patients treated (one patient with bilateral disease/RT)

- Two patients were planned to receive 55 Gy in 22 fractions and 70 Gy in 35 fractions, but did not complete their courses due to cancer-related mortality following two and seven fractions, respectively.

Table 2 summarizes dosimetric parameters. Nearly all institutional target coverage and OAR planning constraints were met; all plans were approved through institutional peer review. In the few cases where constraints were not met, the reason was extensive PTV overlap with one or more OAR (either accepting a minor variation in OAR constraint, or less commonly, accepting lesser PTV coverage when overlapping with spinal cord). 


\section{Cureus}

\section{Variable}

PTV

D95

V110

Lungs-IGTV

V5

V20

Mean (Gy)

Esophagus

V60

Mean (Gy)

Heart

D0.03cc (Gy)

Mean (Gy)

Spinal cord

D0.03cc (Gy)

Left brachial plexus

D0.03cc (Gy)

Right brachial plexus

D0.03cc (Gy)
$0(0-10.3)$

Value (\%) (median, range)

96.6 (93.5 - 100.5)

$0(0-0.3)$

$54.6(19.9-80.1)$

$26.7(4.7-36.9)$

$14.9(5.0$ - 19.4)

$2.0(0-45.2)$

$18.7(4.3-41.6)$

$61.9(16.5$ - 75.6)

10.8 (2.6 - 21.0)

36.2 (5.4 - 48.8)

$0(0-61.6)$

\section{TABLE 2: Dosimetric parameters of targets and organs-at-risk}

Abbreviations: PTV, planning target volume; D95, dose received by $95 \%$ of a structure; V110, volume receiving $110 \%$ of the prescription dose; IGTV, internal gross tumor volume; V5, volume receiving 5 Gy; Gy, Gray; V20, volume receiving 20 Gy; V60, volume receiving 60 Gy; D0.03cc, dose received by 0.03 cubic centimeters of a structure

\section{Initial disease-control outcomes}

There were seven deaths (23\%), which occurred at a median of 5.9 months (range, 0.3-13.8 months) from start of RT: five (71\%) due to progressive LC (predominantly from distant, systemic failure) and two (29\%) due to intercurrent causes (one from infection, and one from pulmonary hypertension). One-year overall survival was $73 \%$. No deaths were RT-related. There were two (6\%) local (both in-field parenchyma), five (16\%) regional (all mediastinal or hilar lymph nodes), and eight (26\%) distant failures. Both local failures occurred in patients who simultaneously experienced regional failures, at 9.0 and 10.9 months from start of RT.

\section{Acute toxicity}

Twenty-nine patients were evaluable for acute toxicity. Acute grade 2 toxicities occurring in $>15 \%$ of patients were fatigue, cough, dysphagia, dermatitis, and esophagitis, in seven (24\%), seven (24\%), six (21\%), six (21\%), and five (17\%) patients, respectively. Acute grade 3 toxicities were fatigue $(3 \%, n=1)$ and dyspnea $(3 \%, \mathrm{n}=1)$. There were no grade $\geqslant 4$ toxicities.

\section{Image-guided radiotherapy couch corrections}

The average ( \pm one standard deviation) translational couch correction magnitude using daily kV CBCT for all fractions of 6X-FFF ORL TRT for all patients was $0.90 \pm 0.50 \mathrm{~cm}$ (Figure 2). 


\section{Cureus}

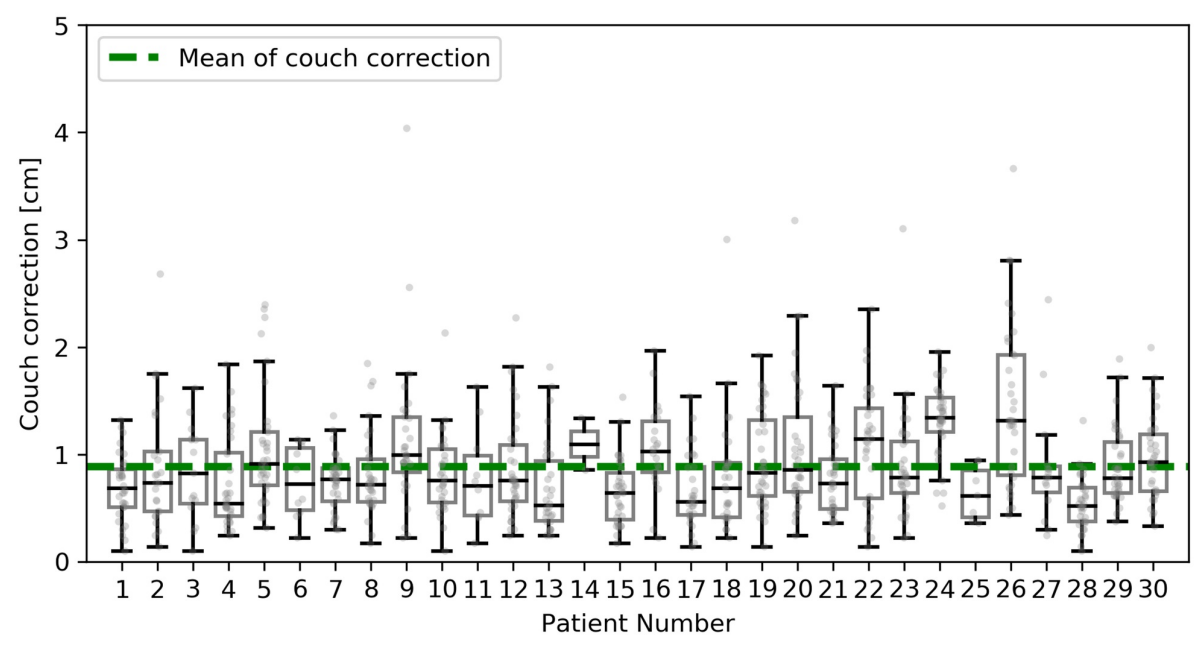

FIGURE 2: Couch corrections after initial setup based on image-guided radiotherapy

Average translational couch correction magnitude using daily kilovoltage cone-beam computed tomography ( $\mathrm{KV}$ CBCT) for all patients, for all fractions of thoracic radiotherapy.

\section{Treatment time and throughput analysis}

Treatment time is depicted in Figure 3 (beam-on and beam-on plus CBCT times) and Figure 4 (total in-room time) for all non-setup fractions. Outlier times were not excluded from average calculations because the reasons for extended treatment times were not routinely recorded (i.e. excluding quantitative outliers without being certain that they were non-linac-related could bias the results in favor of shorter treatment times if the prolonged times were indeed linac-related). The average beam-on and beam-on plus CBCT times for all patients were $1.7 \pm 1.1 \mathrm{~min}$, and $5.0 \pm 3.2 \mathrm{~min}$, respectively. The average beam-on and beam-on plus CBCT times for patients treated with VMAT $(1.3 \pm 0.6 \mathrm{~min}$ and $4.6 \pm 2.8 \mathrm{~min}$, respectively) were significantly shorter than those for patients treated with static-field IMRT ( $2.1 \pm 1.4 \mathrm{~min}$ and $5.5 \pm 3.6 \mathrm{~min}$, respectively, p's $<0.05$ ). The average total in-room time (from gowning area to linac back to gowning area) for all patients was $16.8 \pm 7.9 \mathrm{~min}$, and did not significantly differ between patients treated with VMAT $(16.5 \pm 8.6 \mathrm{~min})$ and static-field IMRT $(17.0 \pm 7.0 \mathrm{~min}, \mathrm{p}=0.39)$.

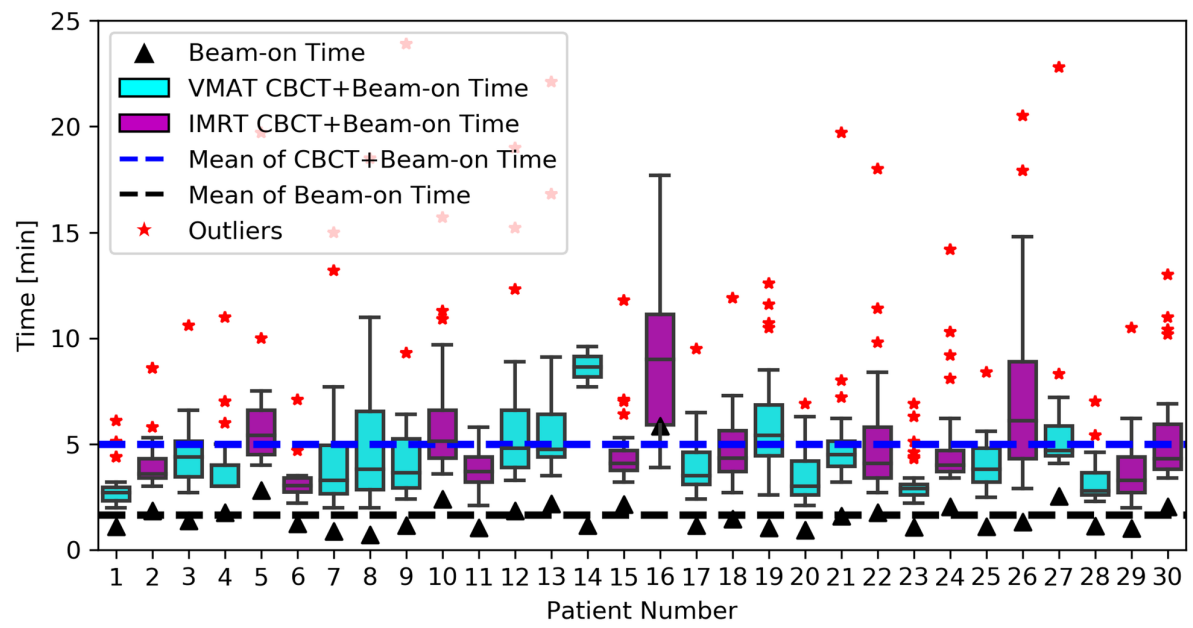

\section{FIGURE 3: Treatment time}

Average beam-on and beam-on plus cone-beam computed tomography (СВCT) times for all patients for all non-setup fractions of $6 \mathrm{MV}$-flattening-filter-free O-ring linear accelerator radiotherapy. Treatment times were significantly shorter for patients who received volumetric-modulated arc therapy (VMAT) versus those who received static-field intensity modulated radiation therapy (IMRT) $(p<0.05)$. 


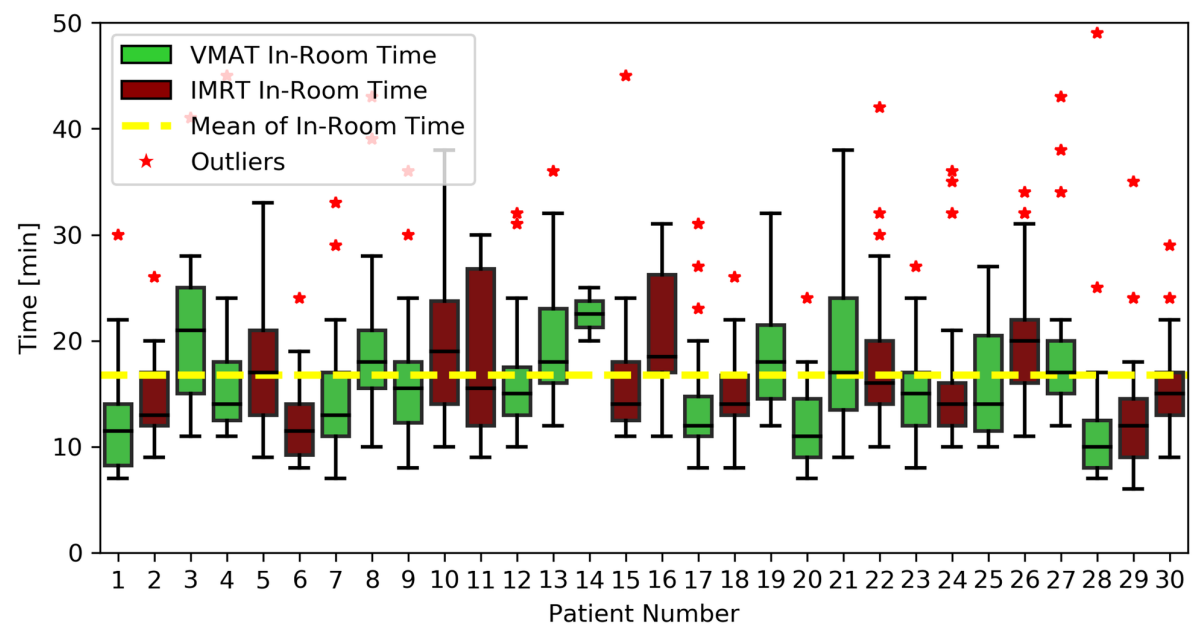

\section{FIGURE 4: Total in-room time}

Average total in-room time (time from leaving gowning area to returning to gowning area) for all non-setup fractions of $6 \mathrm{MV}$-flattening-filter-free O-ring linear accelerator radiotherapy.

Abbreviations: VMAT, volumetric-modulated arc therapy; IMRT, intensity-modulated radiation therapy

\section{Discussion}

Herein, we share the initial clinical experience delivering TRT for patients with LC on a 6X-FFF ORL, which is also the largest published clinical experience of 6X-FFF ORL use to date. We demonstrated the varied clinicopathologic scenarios, initial disease-control outcomes, acute toxicity, dosimetry, couch corrections, and treatment speed of 6X-FFF ORL TRT in LC. The linac was able to deliver TRT to patients with various LC histologies, stages, tumor locations (including bilateral), simulation/setup techniques, and dosefractionation regimens, indicating that it is capable of delivering IMRT to patients with LC in most clinical scenarios.

We found that TRT planning for LC on a 6X-FFF ORL achieved nearly all institutional dosimetric target and OAR constraints, with the exception of a small number of select, unavoidable circumstances where there was overlap between the PTV and OARs, warranting minor variations in the OAR constraint to maximize target coverage, or PTV coverage to spare spinal cord, at the discretion of the treating physician. All plans were approved via institutional peer review.

IGRT for 6X-FFF ORL TRT with daily CBCT offered acceptable average 3D vector couch correction based on СВCT guidance for all patients $(0.89 \pm 0.50 \mathrm{~cm})$. This compares well with prior reported average 3D vector couch correction values for TRT for LC, which ranged from $0.53-1.14 \mathrm{~cm}[25,26]$. Yeung et al. found an average 3D vector couch correction of $0.53 \mathrm{~cm}$, calculated by computing the square root of the sum of the squares of the couch corrections utilizing data from daily $\mathrm{kV} \mathrm{CBCT}$ in each of the left-right, superiorinferior, and anterior-posterior dimensions [25]. Higgins et al. found an average 3D vector couch correction of $1.14 \mathrm{~cm}$, utilizing data from daily CBCT (unspecified whether MV or kV CBCT) with match to carina in patients receiving TRT with parallel opposed fields, 3D-CRT, or IMRT [26]. While statistical comparison between our report and the published studies was not feasible given specific differences between the studies, the couch correction values were qualitatively similar.

The average beam-on, beam-on plus CBCT, and total in-room times for all non-setup fractions were $1.7 \pm 1.1$ $\min , 5.0 \pm 3.2 \mathrm{~min}$, and $16.8 \pm 7.9 \mathrm{~min}$, respectively. The beam-on times observed compared well with previously published beam-on time values for fractionated TRT for NSCLC [27,28]. Mukai et al. reported beam-on times of $4.5 \pm 1.3 \mathrm{~min}$ and $9.8 \pm 1.5 \mathrm{~min}$ to deliver IMRT for NSCLC using TomoDirect and TomoHelical systems, respectively [27]. Shrimali et al. reported median beam-on times of $2.6 \mathrm{~min}$ and 3.0 min for thoracic VMAT and static-field IMRT, respectively ( $\mathrm{p}=0.03)$, for NSCLC [28]. Thus, the beam-on times observed for 6X-FFF ORL TRT for NSCLC compared favorably with those of thoracic IMRT on other linacs, even with patients receiving treatment with as many as five arcs in our study. Like Shrimali et al., we observed that beam-on times were significantly shorter for VMAT treatments as compared to static-field IMRT $(1.3 \pm 0.6 \mathrm{~min}$ vs. $2.1 \pm 1.4 \mathrm{~min}, \mathrm{p}<0.05)$, which may be explained by VMAT’s high beam delivery efficiency from dynamic dose delivery and restricted MLC motion per gantry degree, requiring less MU to deliver the same dose as static-field IMRT $[28,29]$. That said, the total in-room times for patients treated with VMAT vs. static-field IMRT did not statistically differ, which may be a more clinically-meaningful endpoint. As such, clinicians need not avoid static-field IMRT plans out of fears of prolonged patient treatment times relative to VMAT, and should choose the modality that delivers the best plan for the individual patient. At 
our institution, in situations where high-speed optimization is required, such as online adaptive replanning, static-field IMRT has been the preferred approach, as the ability to deliver IMRT as fast as VMAT allows the focus to remain on plan quality and clinical indication, as opposed to efficiency trade-offs. To the best of our knowledge, no published beam-on plus CBCT or total in-room time data for TRT exist. As a means of comparison, mean beam-on plus CBCT and total in-room times for 6X-FFF ORL breast RT (4.4 \pm 0.4 min and $12.4 \pm 0.5 \mathrm{~min}$, respectively) [20] and pelvic RT (3.6 $\pm 0.4 \mathrm{~min}$ and $10.8 \pm 1.4 \mathrm{~min}$, respectively) are provided [12]. While the total in-room time qualitatively appears longer than the breast and pelvic experiences, it is important to note that those experiences excluded prolonged outlier treatment times that were due to nonlinac-related externalities including physician availability for on-board imaging approval, transport staff availability, bladder-filling troubleshooting, and interlock issues requiring physics override. In the pelvic experience, 42 of the 127 time values were excluded for such reasons. In the current experience, outlier times were not excluded, because reasons for prolonged treatments were not recorded. Consequently, we felt that the most conservative way to report the data would be to include all data points, even if it was likely that there were explanations external to the 6X-FFF ORL that accounted for many of the prolonged times.

While prior reports have shown short treatment times and high throughput for patients treated on a 6X-FFF ORL for breast cancer [20], gynecologic cancers [12], and MPM [21], our report is the first clinical report of TRT using this linac for LC, and showed similar results. Quick treatments may improve the patient experience, as treatment tables are often uncomfortable, and maintaining immobility for prolonged periods of time may be difficult, particularly in patients with LC, many of whom may be elderly, frail, and/or have underlying pulmonary and cardiovascular co-morbidities. A high-throughput linac may also help reduce patient wait times and reduce departmental staffing demands.

This report has several limitations. Further follow-up is needed to assess late toxicity and long-term outcomes. As previously discussed, outlier times were not excluded, and therefore our total in-room time values may be longer than the true in-room times for 6X-FFF ORL TRT. This study reports on fractionated TRT for locally-advanced or metastatic LC; stereotactic RT techniques for TRT on a 6X-FFF ORL are beyond the scope of this report, but warrant study. Prospective study of TRT on the 6X-FFF ORL, particularly in comparison to TRT on a C-arm linac, is also a potential direction for future study.

\section{Conclusions}

Herein, we report the initial published clinical experience of 6X-FFF ORL TRT for LC. We showed the linac's versatility with respect to clinical patient and setup variety, adequacy with respect to dosimetry and couch corrections, and fast treatment times relative to those of CALs. As such, TRT on a 6X-FFF ORL may improve departmental throughput without a detriment in radiotherapy quality.

\section{Additional Information \\ Disclosures}

Human subjects: Consent was obtained by all participants in this study. IRB of the University of Pennsylvania issued approval 831888. This clinical research was undertaken with IRB approval from the IRB of the University of Pennsylvania (approval number 831888). Animal subjects: All authors have confirmed that this study did not involve animal subjects or tissue. Conflicts of interest: In compliance with the ICMJE uniform disclosure form, all authors declare the following: Payment/services info: All authors have declared that no financial support was received from any organization for the submitted work. Financial relationships: Abigail T. Berman declare(s) personal fees from Varian Medical Systems. Speaker (unrelated to this work). Lei Dong declare(s) a grant and personal fees from Varian Medical Systems. Grant and personal fees are unrelated to this work. James M. Metz declare(s) a grant, personal fees and Advisory board from Varian Medical Systems. Advisory board, grant funding, and personal fees, all unrelated to this work. Other relationships: All authors have declared that there are no other relationships or activities that could appear to have influenced the submitted work.

\section{Acknowledgements}

Dr. Taoran Li and Dr. Steven Feigenberg contributed equally to this work, as co-senior authors.

\section{References}

1. Ettinger DS, Wood DE, Chair V, et al.: NCCN Guidelines, Version 3.2020: Non-Small Cell Lung Cancer . National Comprehensive Cancer Network, 2020.

2. Kishor Ganti AP, Loo BW, Chair V, et al.: NCCN Guidelines Version 3.2020: Small Cell Lung Cancer . National Comprehensive Cancer Network, 2020.

3. Videtic GM, Donington J, Giuliani M, et al.: Stereotactic body radiation therapy for early-stage non-small cell lung cancer: executive summary of an ASTRO evidence-based guideline. Pract Radiat Oncol. 2017, 7:295-301. 10.1016/j.prro.2017.04.014

4. Faivre-Finn C, Snee M, Ashcroft L, et al.: Concurrent once-daily versus twice-daily chemoradiotherapy in patients with limited-stage small-cell lung cancer (CONVERT): an open-label, phase 3, randomised, superiority trial. Lancet Oncol. 2017, 18:1116-1125. 10.1016/S1470-2045(17)30318-2

5. Antonia SJ, Villegas A, Daniel D, et al.: Durvalumab after chemoradiotherapy in stage III non-small cell lung 
cancer. N Engl J Med. 2017, 377:1919-1929. 10.1056/NEJMoa1709937

6. Aupérin A, Le Péchoux C, Rolland E, et al.: Meta-analysis of concomitant versus sequential radiochemotherapy in locally advanced non - small-cell lung cancer. J Clin Oncol. 2010, 28:2181-2190. 10.1200/JCO.2009.26.2543

7. Turrisi AT, Kim K, Blum R, et al.: Twice-daily compared with once-daily thoracic radiotherapy in limited small-cell lung cancer treated concurrently with cisplatin and etoposide. N Engl J Med. 1999, 340:265-271. 10.1056/NEJM199901283400403

8. Gomez DR, Tang C, Zhang J, et al.: Local consolidative therapy vs. maintenance therapy or observation for patients with oligometastatic non-small-cell lung cancer: long-term results of a multi-institutional, phase II, randomized study. J Clin Oncol. 2019, 37:1558-1565. 10.1200/JCO.19.00201

9. Slotman BJ, Van Tinteren H, Praag JO, et al.: Use of thoracic radiotherapy for extensive stage small-cell lung cancer: a phase 3 randomised controlled trial. Lancet. 2015, 385:36-42. 10.1016/S0140-6736(14)61085-0

10. McAvoy S, Ciura K, Wei C, et al.: Definitive reirradiation for locoregionally recurrent non-small cell lung cancer with proton beam therapy or intensity modulated radiation therapy: predictors of high-grade toxicity and survival outcomes. Int J Radiat Oncol Biol Phys. 2014, 90:819-827. 10.1016/j.ijrobp.2014.07.030

11. Chun SG, Hu C, Choy H, et al.: Impact of intensity-modulated radiation therapy technique for locally advanced non-small-cell lung cancer: a secondary analysis of the NRG oncology RTOG 0617 randomized clinical trial. J Clin Oncol. 2017, 35:56-62. 10.1200/JCO.2016.69.1378

12. Barsky AR, Kim MM, Dreyfuss A, et al.: Initial clinical experience treating patients with gynecologic cancers on a 6MV flattening filter free O-ring linear accelerator. Adv Radiat Oncol. 2020, 10.1016/j.adro.2020.01.004

13. Cashmore J: The characterization of unflattened photon beams from a 6 MV linear accelerator . Phys Med Biol. 2008, 53:1933-1946. 10.1088/0031-9155/53/7/009

14. Michiels S, Poels K, Crijns W, et al.: Volumetric modulated arc therapy of head-and-neck cancer on a fastrotating O-ring linac: plan quality and delivery time comparison with a C-arm linac. Radiother Oncol. 2018, 128:479-484. 10.1016/j.radonc.2018.04.021

15. Li T, Scheuermann R, Lin A, et al.: Impact of multi-leaf collimator parameters on head and neck plan quality and delivery: a comparison between Halcyon(TM) and Truebeam ${ }^{\circledR}$ treatment delivery systems. Cureus. 2018, 10:3648. 10.7759/cureus.3648

16. O'Grady F, Barsky AR, Anamalayil S, et al.: Increase in superficial dose in whole-breast irradiation with Halcyon straight-through linac compared with traditional C-arm linac with flattening filter: in vivo dosimetry and planning study. Adv Radiat Oncol. 2020, 5:120-126. 10.1016/j.adro.2019.07.011

17. Petroccia HM, Malajovich I, Barsky AR, et al.: Spine SBRT with Halcyon(TM): Plan quality, modulation complexity, delivery accuracy, and speed. Front Oncol. 2019, 9:1-8. 10.3389/fonc.2019.00319

18. Li T, Irmen P, Liu H, et al.: Dosimetric performance and planning/delivery efficiency of a dual-layer stacked and staggered MLC on treating multiple small targets: a planning study based on single-isocenter multitarget stereotactic radiosurgery (SRS) to brain metastases. Front Oncol. 2019, 9:7. 10.3389/fonc.2019.00007

19. Bollinger D, Laugeman E, Li T, et al.: Technical note: dosimetric characterization of the dynamic beam flattening MLC sequence on a ring shaped, jawless linear accelerator with double stacked MLC. Med Phys. 2020, 47:948-957. 10.1002/mp.14001

20. Barsky AR, O'Grady F, Kennedy C, et al.: Initial clinical experience treating patients with breast cancer on a 6-MV flattening-filter-free O-ring linear accelerator. Adv Radiat Oncol. 2019, 4:571-578. 10.1016/j.adro.2019.05.006

21. Barsky AR, Kim MM, Maxwell R, et al.: Initial clinical experience treating patients with palliative radiotherapy for malignant pleural mesothelioma on the Halcyon(TM) linear accelerator. Ann Palliat Med. 2020, 10.21037/apm-20-385

22. Le Pechoux C, Dunant A, Pignon JP, et al.: Phase III study comparing post-operative conformal radiotherapy to no post-operative radiotherapy in patients with completely resected non-small cell lung cancer and mediastinal N2 involvement (Lung ART). Elsevier Science, Amsterdam; 2010.

23. Van Esch A, Tillikainen L, Pyykkonen J, et al.: Testing of the analytical anisotropic algorithm for photon dose calculation. Med Phys. 2006, 33:4130-4148. 10.1118/1.2358333

24. Virtanen P, Gommers R, Oliphant TE, et al.: SciPy 1.0: fundamental algorithms for scientific computing in Python. Nat Methods. 2020, 17:261-272. 10.1038/s41592-019-0686-2

25. Yeung AR, Li JG, Shi W, et al.: Tumor localization using cone-beam CT reduces setup margins in conventionally fractionated radiotherapy for lung tumors. Int J Radiat Oncol Biol Phys. 2009, 74:1100-1107. 10.1016/j.ijrobp.2008.09.048

26. Higgins J, Bezjak A, Franks K, et al.: Comparison of spine, carina, and tumor as registration landmarks for volumetric image-guided lung radiotherapy. Int J Radiat Oncol Biol Phys. 2009, 73:1404-1413. 10.1016/j.ijrobp.2008.06.1926

27. Mukai Y, Omura M, Hashimoto H, et al.: Treatment outcome for locally advanced non-small-cell lung cancer using TomoDirect plan and its characteristics compared to the TomoHelical plan. J Med Radiat Sci. 2018, 65:55-62. 10.1002/jmrs.265

28. Shrimali R, Arunsingh M, Reddy G, et al.: Actual gains in dosimetry and treatment delivery efficiency from volumetric modulated arc radiotherapy for inoperable, locally advanced lung cancer over five-field forwardplanned intensity-modulated radiotherapy. Indian J Cancer. 2017, 54:155. 10.4103/ijc.IJC_79_17

29. Davidson MT, Blake SJ, Batchelar DL, et al.: Assessing the role of volumetric modulated arc therapy (VMAT) relative to IMRT and helical tomotherapy in the management of localized, locally advanced, and postoperative prostate cancer. Int J Radiat Oncol Biol Phys. 2011, 80:1550-1558. 10.1016/j.ijrobp.2010.10.024 